matorio que nos aleja de la elegante y acompasada manera de narrar del padre. A pesar de la atmósfera crepuscular que impregna la escena, Carletto no oscurece su paleta, con lo que confiere al cuadro una cierta extraña luminosidad. Asimismo, se vale de un cierto claroscurismo de raíz bassanesca. Con esta obra demuestra nuestro pintor algo de lo que ha aprendido de su padre y del taller de los Bassano: de aquél a componer con cierta majestad y grandeza y de éste a poner más al alcance del espectador lo representado.

José María Ruiz Manero

Doctor en Historia del Arte

\title{
HALLAZGO DEL PRIMER CUADRO EXPUESTO POR RAIMUNDO DE MADRAZO
}

Queremos presentar en este trabajo un cuadro del pintor Raimundo de Madrazo sobre el que la bibliografía del artista ha hecho mención en numerosas ocasiones, por tratarse del primer cuadro que dicho artista presentó en una exposición; fue realizado a la temprana edad de dieciséis años. Es un óleo sobre lienzo, de $130 \times 221$ centímetros firmado en el ángulo inferior izquierdo: «R. Madrazo/1859». Representa La traslación de los restos del apóstol Santiago a la sede de Padrón. El cuadro fue adquirido en 1906 en Sevilla, y desde entonces ha permanecido en la misma colección particular. Se conserva un documento que certifica el origen del mismo y se pagaron 1.000 pesetas por su importe. En el momento de su compra, los profesores de la Escuela de Bellas Artes de Sevilla, Fernando Tirado y Francisco Anaya y León ratificaron la autoría de mano de Raimundo de Madrazo, al igual que el pintor Alfonso Cañaveral, que además añade el dato de que Cecilia de Madrazo posó para la figura del ángel y que el mismo Fortuny pintó el personaje que aparece en el fondo levantando los brazos. También ratifica la autenticidad de la firma J. García y Ramos, que al rubricar los apellidos con la sola inicial de su nombre, nos plantea la interrogante de saber si se trata de José o de su hermano Juan. Nos inclinamos a pensar que es Juan, porque no adorna su nombre con los títulos de presidente de la Academia Libre de Bellas Artes (nombramiento que se le hizo tras su vuelta del extranjero en 1881) ${ }^{1}$ o el de académico de Bellas Artes (que disfrutó José desde 1893) ${ }^{2}$; además, si comparamos la rúbrica del documento con alguna de las que se aprecian en reproducciones de sus cuadros podemos notar diferencias. Y nos consta por otro documento, conservado en la misma colección, que dos años antes de la compra del lienzo de Madrazo se habían adquirido otros cuadros en Sevilla, concretamente ocho cobres flamencos que fueron comprados en 1904 a don Juan García y Ramos, lo que demuestra una relación comercial de continuidad de este pintor con el mismo coleccionista.

En el mismo certificado de 1906 el restaurador Manuel Fatuarte ratifica el perfecto estado de conservación de la obra. Siendo bien conocidos en el ámbito sevillano tres de los firmantes de este documento, Fernando Tirado, Alfonso Cañaveral y García Ramos, no tenemos datos acerca de Francisco Anaya y León ni de Manuel Fatuarte, aunque de éste sí sabemos de sus labores de restauración y valoraciones para la misma colección e incluso se conserva en ella una pequeña obra original suya, una vista arquitectónica por él rubricada.

\footnotetext{
1 Quesada, L., La vida cotidiana en la pintura andaluza, Focus, Sevilla, 1992, p. 196.

${ }^{2}$ Valdivieso, E., Historia de la pintura sevillana. Siglos xIII al XX, Guadalquivir, Sevilla, 1986, p. 427.
} 
El cuadro de La traslación de los restos de Santiago a la sede de Padrón es de dimensiones considerables; muestra las figuras dispuestas siguiendo un esquema elíptico, en variadas posturas, marcando una diagonal dominante el sudario que envuelve el cuerpo del apóstol, continuado por los brazos y alas del ángel. Sirve de escenario al conjunto de personajes el hermoso paisaje de ría gallega, que eleva el nivel del horizonte, creando un amplio espacio pictórico. Al fondo se ven varios rebaños paciendo. Los protagonistas descienden de una embarcación que cierra el espacio por el extremo izquierdo. El lienzo muestra una magnífica realización dibujística, muy detallada, y una concepción general imbuida de las formas solemnes de la pintura histórica todavía en boga. La gama de color resulta variada y rica, creando una mancha intensa de luz las figuras del ángel y del santo vestidos de blanco. También resultan muy atractivas cromáticamente las figuras de los tres personajes infantiles que abren el cortejo (Fig. 1).

El cuadro definitivo tuvo un boceto previo que pertenece hoy a la colección de la Hispanic Society of America, donde pudiera haber ingresado en el año 1913 con el conjunto de obras que el artista donó a esta importante institución cultural; la Hispanic fue fundada en 1904 por el escritor e hispanista Archer Milton Huntigton, con quien Raimundo mantuvo estrechas relaciones, siendo su asesor para la compra de obras destinadas a la importante pinacoteca de esta entidad, de la cual también fue nombrado miembro en $1905^{3}$ (Fig. 2).

El boceto mide $21,5 \times 26,5 \mathrm{cms}$. En él ya se recortan los ángulos superiores del lienzo, creando esquinas curvas. Podemos apreciar algunas variaciones en la disposición de las figuras del cuadro definitivo frente al boceto original. El personaje que sujeta las piernas del apóstol se vuelve hacia el ángel que sostiene la cabeza del mismo, mientras que en el boceto originario dirigía la mirada al suelo. También la figura que está arrodillada en primer plano cambia la orientación de su gesto para contemplar el desembarco. Por otra parte, un anciano sustituye a otro personaje masculino más joven que estaba situado tras la figura de los brazos levantados que encabeza el cortejo.

La celebración del centenario de la muerte de Federico de Madrazo ha propiciado una revisión bibliográfica importante sobre el padre de nuestro artista, lo que a su vez ha supuesto también la aparición de notables aportaciones sobre Raimundo ya que ahora se han visto publicadas abundantes noticias procedentes del archivo familiar de los Madrazo. Era conocida la existencia de una gran cantidad de documentación acerca de esta saga, destacándose las minuciosas agendas de Federico, en las que solía detallar sus actividades y en las que reflejaba valiosos datos sobre el ambiente artístico del momento ${ }^{4}$.

El cuadro de La traslación del cuerpo de Santiago a la sede de Padrón es, como ya se ha indicado, obra mencionada abundantemente por la bibliografía que se ha ocupado del pintor, pero ha permanecido inédita hasta ahora su reproducción y estudio. Se nos planteaban algunas interrogantes a la vista de la rúbrica de la fecha y de las palabras de Cañaveral en el documento que acompañó a su compra. Se menciona como fecha de su exhibición en concurso la de 1858 , si bien el cuadro aparece signado en $1859^{5}$. Por tanto, hay que plantear que el autor fir-

\footnotetext{
${ }^{3}$ González López, C. y Martí Ayxela, M., Raimundo de Madrazo, un pintor español en París, Caja Rural del Jalón, Zaragoza, 1996, pp. 49-50.

${ }^{4}$ González López, C., «El hogar de Fortuny», Revista de Ideas Estéticas n. ${ }^{\circ}$ 131, C.S.I.C., Madrid, 1975, pp. $213-229$. En este artículo (nota $n .^{\circ} 13$ ) se menciona la existencia de unas Memorias inéditas de Raimundo Madrazo, sin foliar, que existen en el archivo de la familia Madrazo. González López, C. y Martí Aixela, M., Catálogo de la exposición Federico de Madrazo (1815-1894), Museo Romántico, Madrid, 1994. VV.AA., Catálogo de la exposición Federico de Madrazo y Kunt (1815-1894), Museo del Prado, Madrid, 1994.

${ }^{5}$ Enciclopedia Universal Ilustrada Espasa-Calpe, T. XXXI, Madrid, 1975, p. 1364. Ossorio y Bernard, M., Galería biográfica de artistas españoles del Siglo XIX, Madrid, 1975, p. 400 (cita erróneamente como fecha de exposición del cuadro 1868). De la Puente, J., «Innovación y conservadurismo en los Madrazos», Revista Goya n. ${ }^{\circ} 104$, Madrid, 1971, p. 104 (cita la presentación del cuadro en Sevilla en 1858). González, C. y Martí, M., Pintores españoles en París (1850-1900), Barcelona, 1989, p. 159. Aunque no citan en concreto la obra, sí la participación del pintor en la exposición sevillana de
} 


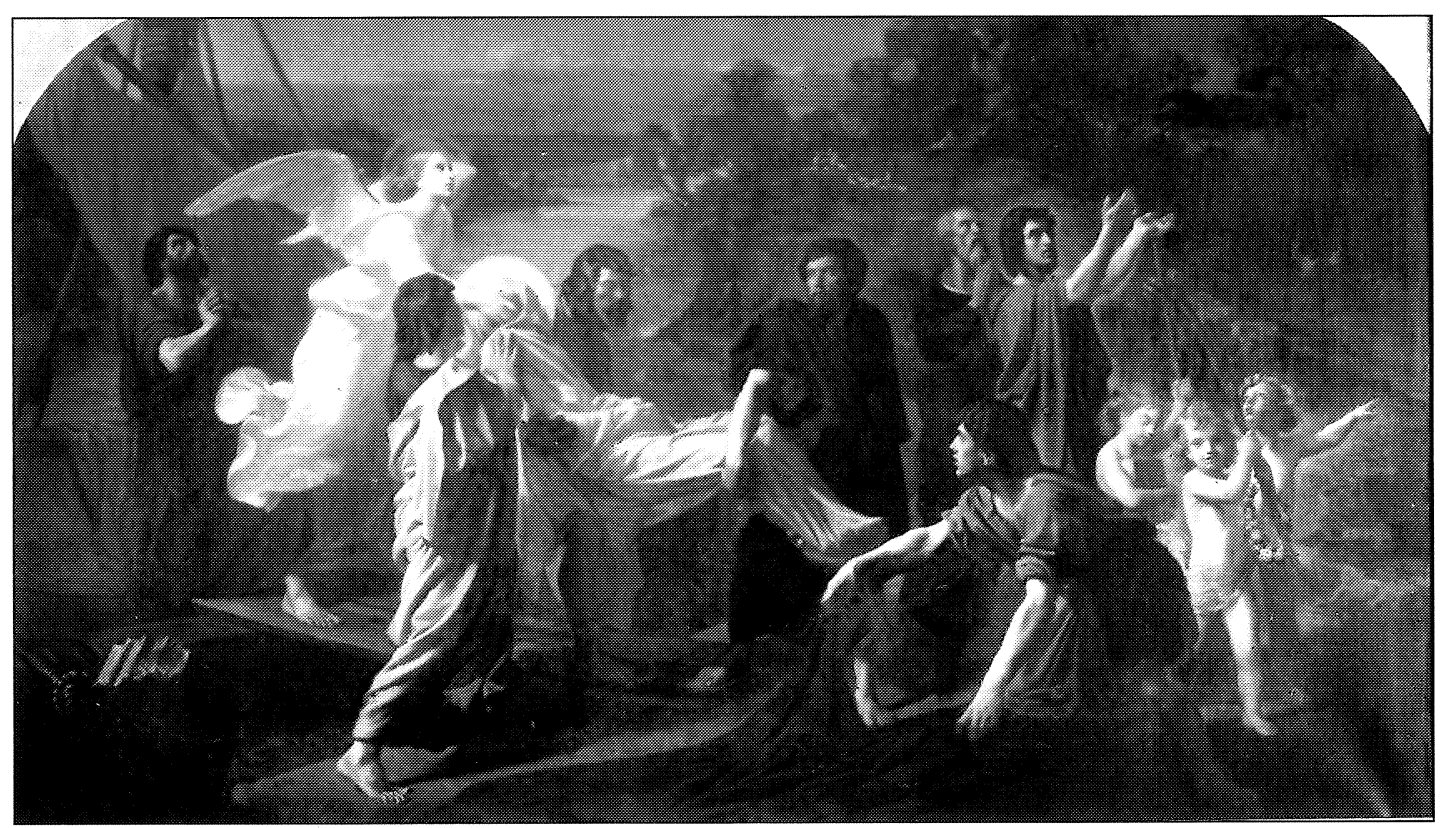

1

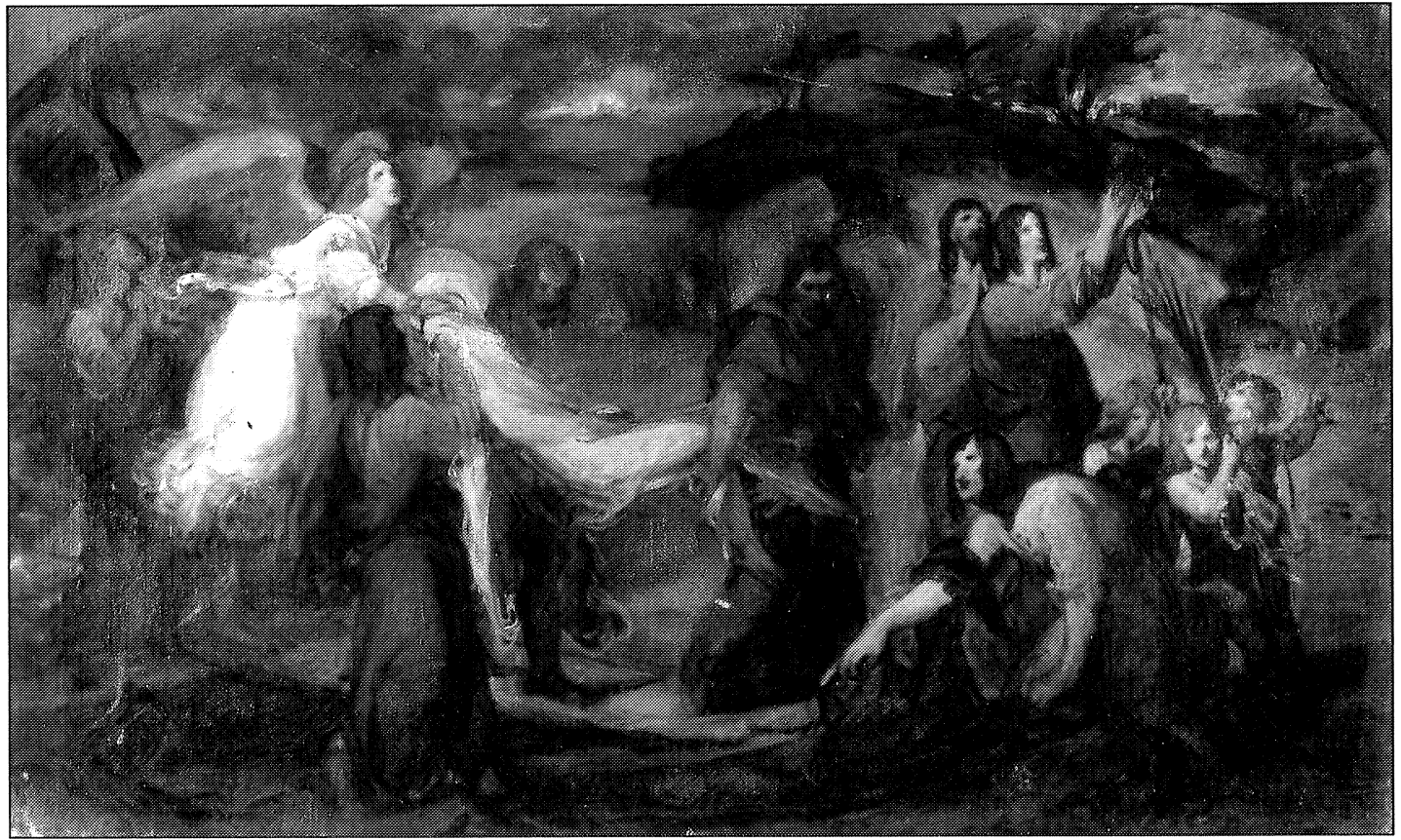

2

Fig. 1. Raimundo de Madrazo y Garreta: La traslación de los restos del Apóstol Santiago a la sede de Padrón. Colección particular.

Fig. 2. Raimundo de Madrazo: Boceto para el cuadro "La traslación de los restos de Santiago a la sede de Padrón» (Courtesy of The Hispanic Society of America). 
mó la obra posteriormente a su realización y exhibición, puesto que los documentos del archivo Madrazo corroboran el año 1858 como el de la participación en la Regional de Bellas Artes de Sevilla. Además, en este año Raimundo estuvo en Sevilla acompañando a su padre en una estancia que duró desde el 8 de octubre hasta el 18 de noviembre ${ }^{6}$. Allí contactaron con el ambiente artístico local y estrecharon sus relaciones con los duques de Montpensier. Raimundo tenía entonces diecisiete años, y estaba estudiando desde los catorce en la Academia de San Fernando. Según cita Martí, el año anterior había pintado el tema de «La traslación de los restos de Santiago a la sede de Padrón» ${ }^{7}$. En Sevilla se dedicó a copiar temas de Murillo en el Museo, estatuas en la catedral y a conocer, junto a su padre, estudios de pintores y colecciones particulares, estableciendo estrechas relaciones, entre otras, con la familia de pintores apellidados Cañaveral.

Por otra parte, Alfonso Cañaveral indica en el documento firmado en 1906, refiriéndose a nuestro cuadro, que sirvió de modelo para la cabeza del ángel la hermana del pintor y posteriormente esposa de Fortuny, Cecilia Madrazo, dato que puede ser totalmente verídico. Lo que no resultaba tan creíble es que la figura que se cita como de mano del catalán, la «que aparece en el fondo del cuadro en actitud de levantar los brazos», fuese realmente pintada por él, puesto que ambos artistas se conocieron en fecha posterior a la de realización del lienzo. González y Martí mencionan como primer encuentro entre ambos pintores el que se realizó en 1865 en la capital francesa. La relación de Fortuny con la familia Madrazo se había iniciado en 1860, cuando el pintor catalán regresaba de Marruecos. Pasa por Madrid y durante su breve estancia fue presentado a Federico Madrazo. Éste le invitó a su estudio y, admirado por los trabajos del joven, expende un permiso para que pudiera copiar en el Museo del Prado ${ }^{8}$. Su matrimonio con Cecilia de Madrazo se celebraría en Madrid en $1866^{9}$.

Pero había que tener en cuenta que el documento que comentamos se fecha casi cincuenta años después de realizada la obra, y podría ser que el pintor Cañaveral tuviese algún conocimiento sobre las colaboraciones de ambos pintores y así lo hubiera querido reflejar en esta certificación, o bien que hubiera alguna intervención posterior de Fortuny en la obra, pero resultaba extraño porque la figura que atribuye Cañaveral al pintor catalán, la que levanta los brazos, sigue muy de cerca el planteamiento del boceto inicial. La reciente monografía sobre el pintor, el catálogo realizado para la exposición celebrada en Zaragoza, con una abundante aportación de datos procedentes del archivo Madrazo, ha venido a aclararnos la cuestión. El tema de la obra fue elegido por Federico de Madrazo, que pretendía que su hijo Raimundo siguiera las pautas temáticas y estilísticas de los nazarenos. El mismo Federico pintó al menos un boceto dedicado también al apóstol Santiago, concretamente la aparición del santo a Ramiro I, cuyo asunto aparece explicado en nota manuscrita al dorso del mismo. La iconografía más habitual de Santiago en la pintura de historia ha sido la que representaba al apóstol interviniendo en la Reconquista de España ${ }^{10}$, aunque también está muy difundida su imagen artística como apóstol y como peregrino ${ }^{11}$. El tema que aquí se nos muestra no resulta frecuente en la

1858. González, C., «Raimundo de Madrazo, un español de París», Antiqvaria n. ${ }^{\circ}$ 18, Madrid, 1985, p. 40. Johnston, W.R., The Nineteenth Century Paintings in the Walters Art Gallery, Baltimore, 1982, pp. 178-9. Valverde Madrid, J., «El pintor Raimundo de Madrazo», Anales de la Real Academia de Bellas Artes de Cádiz, n. ${ }^{\circ}$, Cádiz, 1986, p. 33. cita que no obtuvo recompensa alguna en la Exposición Regional de 1858 de Sevilla con el gran cuadro de historia del apóstol Santiago.

${ }^{6}$ Martí Ayxelá, M., «Federico de Madrazo en Sevilla, 1858», Laboratorio de arte, n. ${ }^{\circ}$ 7, Universidad de Sevilla, Sevilla, 1994, pp. 201-216.

7 Idem, p. 207.

8 González, C. y Martí, M., Mariano Fortuny Marsal, T. I, Barcelona, 1989, p. 36.

${ }^{9}$ González, C., «El hogar de Fortuny», Revista de ideas estéticas, n. ${ }^{\circ} 131$, Madrid, 1975, pp. 213-229.

10 VV.AA., Catálogo de la exposición Federico de Madrazo y Kuntz (1815-1894), Museo del Prado, Madrid, 1994, pp. 175-6.

$"$ Reau, L., Iconographie de l'Art Chrétien, T. III,2 (París, 1958), Nueva York, 1983, pp. 695 y ss. 
pintura española. La creencia del traslado maravilloso del cuerpo del apóstol a Galicia es un episodio legendario que figura en un documento apócrifo del siglo Ix atribuido al Papa León. Hay otra versión en el Codex Calixtinus o Liber Sancti Jacobi, que refiere que después de muerto el apóstol los discípulos colocaron el cuerpo en una nave que, guiada por un ángel, llegó a las costas gallegas ${ }^{12}$.

El joven Raimundo empezó a trabajar en el tema en noviembre de 1856, finalizando el cuadro en 1857 y presentándolo efectivamente en la Exposición Regional de Sevilla en $18588^{13}$. Pero según anotación de la agenda de Federico, de fecha 28 de marzo de 1868, el cuadro fue retocado entonces durante una estancia de Raimundo en Madrid, y fue adquirido por Cañaveral. Por estas fechas ya Fortuny y Raimundo compartían horas de trabajo y ocio, dedicándose a la pintura por las mañanas y dejando las tardes a sus dos aficiones favoritas, los toros y los establecimientos de antigüedades, en los que adquirían piezas para sus colecciones ${ }^{14}$. Así pues no resulta extraño que se diera en este cuadro la colaboración que cita Cañaveral en el documento, y que Fortuny rehiciera o retocara en parte la figura de los brazos en alto, pues es bien sabido que a ambos cuñados les gustaba colaborar juntos en algunas obras.

Por tanto estamos ante una obra destacada en la trayectoria profesional de este pintor del cual se están realizando ahora importantes revisiones bibliográficas que nos acercan a la vida y obra de un artista español que fue prontamente reconocido en ambientes sociales y culturales del extranjero.

\section{Documento que acompañó a la compra del cuadro en el año 1906}

El cuadro cuya fotografía va adjunta firmado «Raimundo Madrazo» es indudablemente de dicho artista. Certifico como discípulo que fui en París de Don Raimundo Madrazo. / Fernando Tirado profesor de la escuela Superior de Bellas Artes de Sevilla.

Doy fé de que la firma de Raimundo de Madrazo que ostenta el cuadro del Apóstol Santiago es auténtica. / Sevilla Abril 1906. J. García y Ramos.

Examinada la importante obra cuya fotografía va adjunta no cabe duda que el autor es Madrazo (D. Raimundo). / El profesor de la escuela de B. Artes / Fran ${ }^{\text {co }}$ Anaya y León.

El cuadro «La traslación milagrosa del cuerpo del apóstol Santiago firmado $R$. Madrazo es por su importancia obra para un Museo Moderno y el cuadro más serio que ha pintado Raimundo Madrazo y lo avaloran interesantes detalles conocidos, que son, haber servido de modelo para la cabeza del celeste mensajero la hermana del autor Cecilia Madrazo mujer que fue del inmortal Fortuny siendo de manos de este insigne artista una figura que aparece en el fondo del cuadro en actitud de levantar los brazos. / Alfonso Cañaveral.

Certifico que el cuadro de Raimundo Madrazo se encuentra en perfecto estado de conservación. / Manuel Fatuarte, Restaurador en Sevilla 1 de Mayo 1906.

\section{M. ${ }^{a}$ Teresa Terrón Reynolds Universidad de Extremadura}

\footnotetext{
${ }_{12}$ Sobre el tema de la predicación de Santiago en España y su desembarco en Galicia ver también el trabajo de Marie de Menaca «Dos problemas diferentes sobre Santiago en España. Su predicación y su sepultura», Actas del Congreso de Estudios Xacobeos, Santiago, 1995, pp. 211-236. Agradecemos a Yolanda Barriocanal López el envío de este trabajo y sus notas sobre la hagiografía del santo.

${ }^{13}$ González López, C. y Martí Ayxelá, M., Raimundo de Madrazo, un pintor español en París, Caja Rural del Jalón, Zaragoza, 1996, p. 12.

${ }^{14}$ Idem, p. 23.
} 